\title{
Magnetophoretic Sorting of Single Catalyst Particles
}

\author{
Miguel Solsona ${ }^{+}$Anne-Eva Nieuwelink ${ }^{+}$, Florian Meirer, Leon Abelmann, Mathieu Odijk, \\ Wouter Olthuis, Bert M. Weckhuysen,* and Albert van den Berg*
}

\begin{abstract}
A better understanding of the deactivation processes taking place within solid catalysts is vital to design better ones. However, since inter-particle heterogeneities are more a rule than an exception, particle sorting is crucial to analyse single catalyst particles in detail. Microfluidics offers new possibilities to sort catalysts at the single particle level. Herein, we report a first-of-its-kind $3 D$ printed magnetophoretic chip able to sort catalyst particles by their magnetic moment. Fluid catalytic cracking (FCC) particles were separated based on their Fe content. Magnetophoretic sorting shows that large Fe aggregates exist within $20 \%$ of the FCC particles with the highest Fe content. The availability of Bronsted acid sites decreases with increasing Fe content. This work paves the way towards a highthroughput catalyst diagnostics platform to determine why specific catalyst particles perform better than others.
\end{abstract}

$R_{\text {ece }}$ with micro-sized structures, such as individual cells and particles. $^{[1-4]}$ The small volumes and high-speed regimes where microfluidics work, allow experiments at the single particle level and in a high-throughput fashion, as well as better control on the parameters affecting the system. ${ }^{[5-10]}$ Different fields have benefitted from microfluidics, however, for catalysis it is far from its full potential. For example, microfluidics has been used for monitoring of chemical reactions ${ }^{[11,12]}$ and catalyst synthesis, offering a better control over temperature and mixing of reagents, resulting in more homogeneously shaped catalysts. ${ }^{[13,14]}$ However, a great potential of microfluidics still lies in the separation of single catalyst particles. Microfluidics offers various techniques to separate catalyst particles based on their physical properties. For example, passive methods, such as pinched flow fractio-

[*] M. Solsona, ${ }^{[+]}$Prof. L. Abelmann, Dr. M. Odijk, Dr. W. Olthuis, Prof. A. van den Berg

BIOS lab on a chip group, MESA + Institute for Nanotechnology

University of Twente

Drienerlolaan 5, Enschede (The Netherlands)

E-mail: a.vandenberg@utwente.nl

A.-E. Nieuwelink, ${ }^{[+]}$Dr. F. Meirer, Prof. B. M. Weckhuysen Inorganic Chemistry and Catalysis, Debye Institute for Nanomaterials Science

Utrecht University

Universiteitsweg 99, 3584 CG Utrecht (The Netherlands)

E-mail: b.m.weckhuysen@uu.nl

Prof. L. Abelmann

KIST Europe

Campus E7, Saarbrücken (Germany)

$\left.{ }^{+}\right]$These authors contributed equally to this work.

(19) Supporting information and the ORCID identification number(s) for iD the author(s) of this article can be found under: https://doi.org/10.1002/anie.201804942. nation and Dean flow fractionation, make use of the inertial properties of particles and fluids in microchannels to sort them by size or shape. On the other hand, active sorting methods make use of external electric, magnetic, optical or acoustic fields to separate particles or cells.

A catalytic system that is very suitable as a showcase for single particle sorting using microfluidics, is the Fluid Catalytic Cracking (FCC) catalyst. ${ }^{[15-17]}$ FCC particles are 50-150 $\mu \mathrm{m}$ sized, multi-component porous particles and responsible for cracking crude oil into gasoline and bulk chemicals, such as propylene. ${ }^{[18,19]}$ During the FCC process, the catalyst deactivates irreversibly due to dealumination and the accumulation of metals, mainly $\mathrm{Fe}, \mathrm{Ni}$, and $\mathrm{V}$. While $\mathrm{Ni}$ and $\mathrm{V}$ decrease the yield by promoting for example, coke formation, $\mathrm{Fe}$ is supposed to deactivate the particles by creating a shell of reduced porosity on the particles' outer surface, either via vitrification of the particle matrix in the presence of Fe and/or co-deposition of silica. ${ }^{[20-29]}$ Therefore, during operation, to create a stable process efficiency, a fraction of catalyst is being replaced on a daily basis, resulting in a mixture called equilibrium catalyst (ECAT). To investigate their deactivation, FCC ECAT particles are often sorted based on their skeletal density, which is associated with metal loading and age. ${ }^{[30]}$ Although the density gradient separationgives a negative correlation between density and catalytic activity, it is a batch process that does not give insight in the individual role of the metals that deactivate FCC particles. Therefore, there is a need for a new method, that is, a tool able to sort ECAT particles by just one of the metals that deactivate them.

Herein, we present for the first time a 3D printed magnetophoretic chip, ${ }^{[31]}$ as outlined in Figure 1 a, which is able to sort individual catalyst particles by their magnetic moment. The sorted FCC particles were subsequently analysed by X-ray fluorescence (XRF) microscopy (Figure 1c), fluorescence micro-spectroscopy (Figure 1d), magnetic hysteresis and field cooled magnetic measurements (Figure 1b) to explain the origin of the sorting principle. Magnetophoresis can be used to sort magnetic and non-magnetic particles and fluids (ferrofluids), ${ }^{[32]}$ and has been previously used to sort cells and magnetic particles. ${ }^{[33-38]}$ This very reproducible approach could also be used for other magnetic catalysts, containing for example, $\mathrm{Ni}$ or Co.

Previous work has found (at least) two types of $\mathrm{Fe}$ distributed within FCC particles: Fe inside the clay components of the catalyst and Fe deposited during the FCC process, which is mainly accumulated in the first $2-3 \mu \mathrm{m}$ of the outer surface of the particle. ${ }^{[18]}$ This deposited Fe, which originates from the feedstock or from reactor debris, is mainly held responsible for the deactivation of these catalysts due to pore blocking. ${ }^{[20,28,29,39]}$ Furthermore, it was shown that these two 


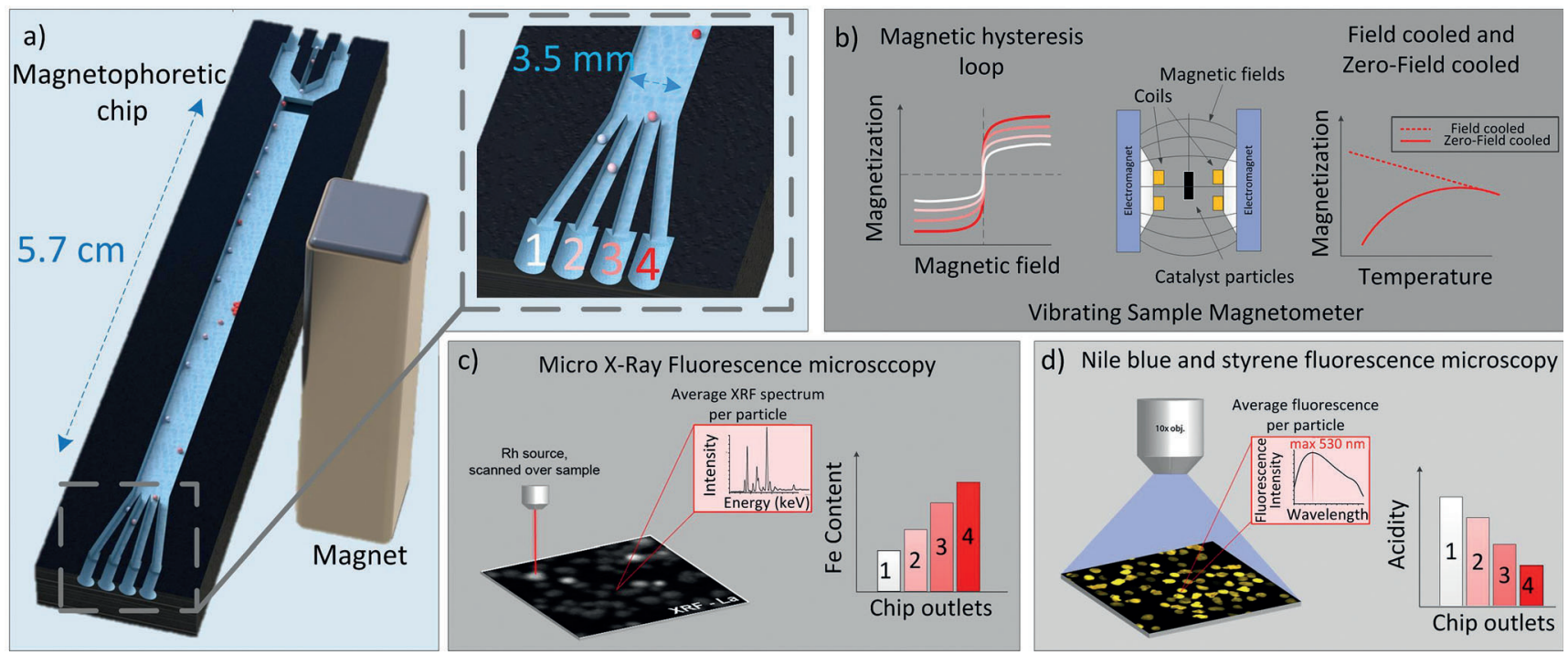

Figure 1. a) Schematic representation of the inside channel of the $3 \mathrm{D}$ printed microfluidic chip for sorting catalyst particles. b) Sketch of the vibrating sample magnetometer, invented hysteresis loops of the different outlets and zero-field cooled and field cooled measurements. c) micro X-ray fluorescence and d) fluorescence micro-spectroscopy to analyse the sorted FCC catalyst particles.

types of $\mathrm{Fe}$ distributions have different oxidation states. Inside the clay, $\mathrm{Fe}$ is found as $\mathrm{Fe}^{0}$ and as $\mathrm{Fe}^{\mathrm{II}}$ in the form of $\mathrm{FeO}$ and $\mathrm{SiFeO}_{3} \cdot{ }^{[28]}$ On the other hand, $\mathrm{Fe}^{\mathrm{III}}$ is found on/in the outer FCC particle layer.

ECAT particles were previously sorted by magnetic fields to decrease the disposal of spent catalyst in FCC reactors. This industrial approach consisted of a permanent rolling magnet cylinder under a belt where particles with different sizes were fed on, to sort them in just two fractions. ${ }^{[40]}$ In this work, however, the particles have been pre-sorted by size and we focus on the relation of $\mathrm{Fe}$ content, magnetic properties and the availability of Brønsted acid sites ${ }^{[41-44]}$ in a wide range of Fe content and on real, industrially processed ECAT particles, providing a unique catalyst diagnostics platform for single particle analysis.

The developed 3D printed microfluidic chip consists of 5 inlets (4 inlets to focus the particles inside the chip and 1 inlet to introduce the particles), a straight channel of $5.7 \mathrm{~cm}$ long, $3.5 \mathrm{~mm}$ wide and $1 \mathrm{~mm}$ deep where the particles get attracted to the magnet, and 4 outlets. Details of the microfluidic chip can be found in Section S1 of the Supporting Information. Considering the purpose of the device to sort by Fe content and knowing that the magnetic force is related to $\mathrm{Fe}$ volume (i.e. total mass of $\mathrm{Fe}$ in the particle), the ECAT particles were size pre-sorted with two sieves in a range or 70 $90 \mu \mathrm{m}$, to have a similar Fe concentration per particle in each fraction. Based on the large differences between magnetic moments found for the ECAT particles, they were sorted into 5 fractions; four microfluidic outlets (Movie S3 in the Supporting Information) and a fifth fraction formed by the particles that adhered to the channel wall at the position of the magnet (Figure S1b, fraction 5).

To know the magnetic properties of the sorted FCC particles, their magnetic moment was measured and the results are shown in Figure 2a. Hysteresis loops per fraction 1 to 5 and averaged per single FCC ECAT particle show a superparamagnetic behaviour, no significant coercivity and an increasing moment per particle with increasing fraction number. This is an indication for either an increase in the amount of magnetic material inside the catalyst particles and/ or a change in form or oxidation state of certain magnetic materials. Consequently, micro-XRF measurements were performed by qualitatively mapping the elemental composition of the ECAT particles in all sorted fractions (Figure S4). Figure $2 \mathrm{~b}$ shows an increased average Fe content in the ECAT particles from fraction 1 to 5 , confirming that $\mathrm{Fe}$ is the main actor on the magnetic attraction to the magnet. Also, when $\mathrm{Fe}$ increases by a factor of 2 the magnetic moment increases by a factor of 13 . This seems to point to a change in form and/or oxidation state. Importantly, Figure S4 shows that all particles have a similar La content ${ }^{[45]}$ (a marker for zeolite US-Y) and thus a similar amount of zeolite per fraction. In FCC deactivation research, $\mathrm{Ni}$ can be used as a measure for the age of an FCC particle, since its concentration increases homogeneously with deactivation of the FCC particles and, in contrast to $\mathrm{Fe}$, is not present in fresh FCC particles. ${ }^{[15,46,47]}$ However, the ECAT under study has a low Ni content, therefore there are no clear trends observed that link our magnetophoretic sorting to particle age.

When taking a closer look at the FCC particles in the five fractions (Figure 3a), aggregates were observed on the surface of the particles of fractions 2 to 5. SEM-EDX measurements (Figure S5) revealed that both black and orange aggregates consist of iron oxide. Electron paramagnetic resonance (EPR) and UV/Vis microscopy data on the aggregates (Figure S6) indicate the presence of $\mathrm{Fe}_{2} \mathrm{O}_{3}$ or $\mathrm{FeO}(\mathrm{OH})$. In Figure $3 \mathrm{~b}$, the percentage of the particles' visible surface (projected to $2 \mathrm{D}$ ) that is covered by these aggregates is listed per fraction. To do this, the images were binarized with a manually set threshold to count the area of the aggregates and particles per 2D image. The percentage of the $2 \mathrm{D}$ visible particle surface that was found covered by 
a)

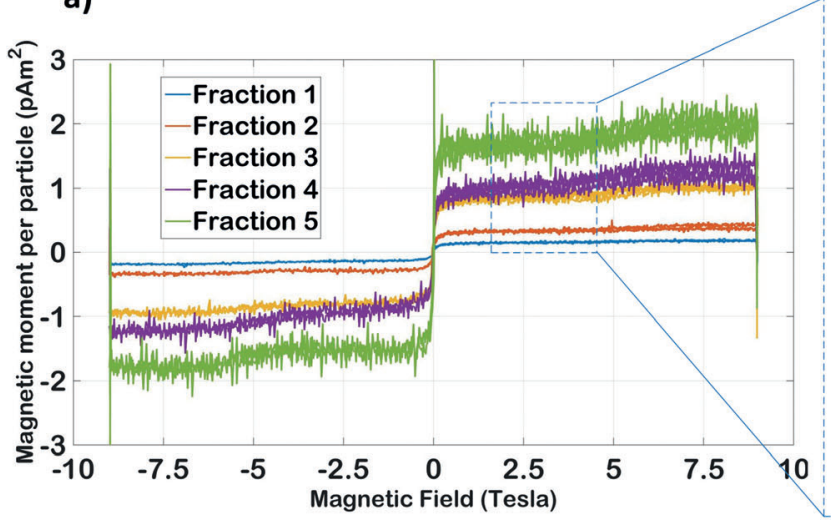

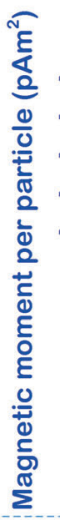

b)

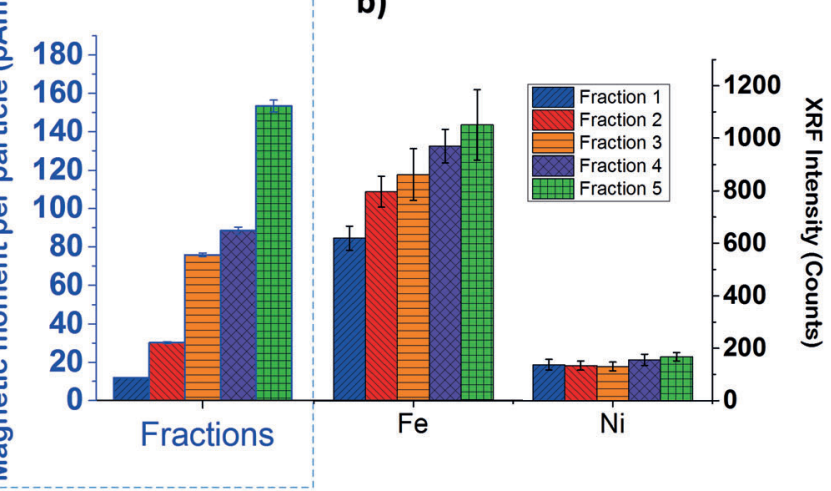

Figure 2. a) Hysteresis loops of the sorted ECAT particles per outlet and per particle. Number of particles per outlet: $1=466,2=255,3=120$, $4=80$ and $5=44$, b) (right axis) relative XRF Fe and Ni intensities and (left axis) magnetic moment per fraction of the sorted single ECAT particle.

a)

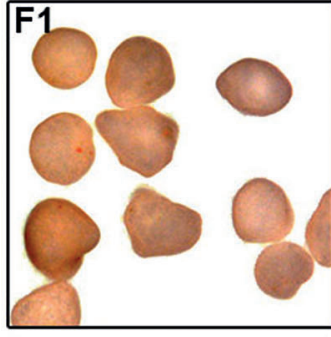

b)

\begin{tabular}{|r|c|c|}
\hline & $\begin{array}{r}\text { area \% with } \\
\text { aggregates }\end{array}$ & \# particles \\
\hline F1 & 0.029 & 200 \\
\hline F2 & 0.030 & 193 \\
\hline F3 & 0.14 & 94 \\
\hline F4 & 0.37 & 149 \\
\hline F5 & 1.4 & 73 \\
\hline
\end{tabular}

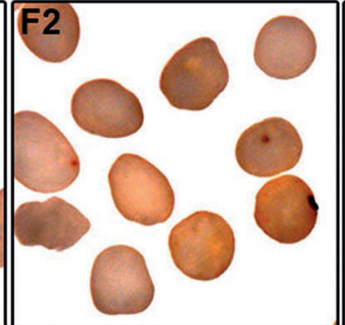

c) Particle distribution
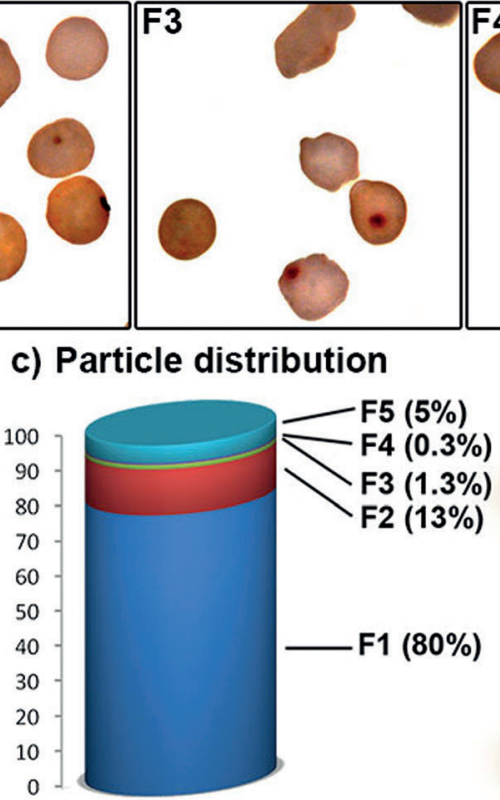

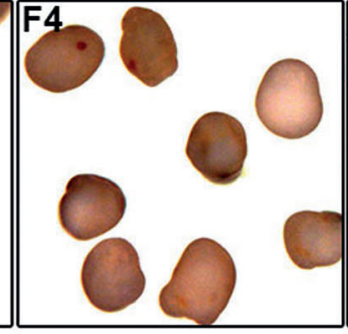

d)
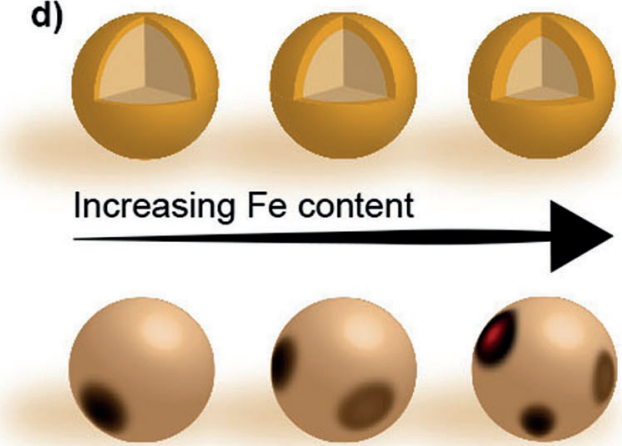
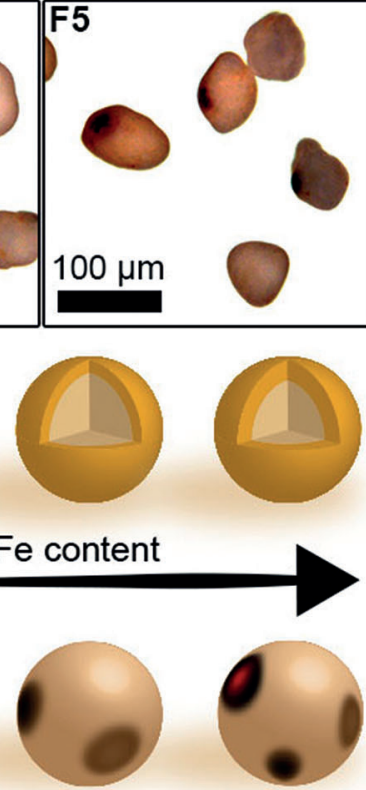

Figure 3. a) Optical images of the sorted ECAT fractions, showing an increasing number of dark spots on their surface. b) Table of the ratio of aggregate area to particle area for each fraction. c) particle distribution of the different fractions Fn. d) the formation of a Fe shell or Fe clusters on the surface of ECAT particles.

black or red aggregates was then calculated showing an increasing covered area from fractions 2 to 5 .

As previously mentioned, it is known that $\mathrm{Fe}$ coming from the crude oil feedstock is deposited mainly in/on the outer rim of the FCC particle, as illustrated in Figure 3 d. $^{[20,24]}$ The FCC catalyst particle distribution over the five fractions (Figure $3 \mathrm{c}$ ) indeed shows that $80 \%$ of the ECAT particles, which are those in fraction 1 , follow this pathway for the deposition of Fe. However, the catalyst particles in fractions 2 to 5, which add up to $20 \%$ of the whole ECAT sample, contain an increasing amount of $\mathrm{Fe}$ aggregates on their surface, as illustrated in Figure $3 \mathrm{~d}$. When magnetic material is present in bigger Fe oxide clusters, the magnetic moment at lower fields is higher. This effect could cause a larger deviation in the Fe content per fraction. For that, a further study on previously shown hysteresis loops by performing a Langevin fit was performed [Eq. (S1) and Section S6]. For further information on the theory on superparamagnetism we refer to Section S2. It was found that there are no big differences between the average cluster sizes of each fraction.

To calculate the range of average cluster sizes inside the ECAT particles and their anisotropy constant, the zero-field cooled (ZFC) and field-cooled (FC) technique were used. With this technique the magnetic moment of a sample is measured when heating with (FC) and without (ZFC) previously applied magnetic field. From the temperature at which the ZFC moment coincides with the FC, which is the blocking temperature $T_{\mathrm{B}}$, and taking $\mu\left(K_{\mathrm{B}} T\right)^{-1}$ from the Langevin fitting, the average cluster size and constant anisotropy can be extracted [Eq. (S5) and (S6)].

Figure 4 presents the ZFC and FC results of fractions 1, 3 and 5 , showing very different behaviours at temperatures 
below $50 \mathrm{~K}$. However, when comparing $T_{\mathrm{B}}$, no significant differences between the fractions are observed. Taking the $T_{\mathrm{B}}$ of fraction 5 as $150 \mathrm{~K}$, we find the ratio of $\mathrm{K}(\mathrm{Ms})^{-1}$ to be 0.23 . This value is 10-times higher than of typical magnetic iron oxides of the form of magnetite and maghemite, 0.04 and 0.02 , respectively. Assuming that in fraction 5 the $\mathrm{Fe}$ content is $3 \mathrm{wt} \%$, similar to the HML ECAT particles from Meirer et al. ${ }^{[23]}$ the anisotropy constant and the saturation magnetization can be calculated (Section S2) resulting in $17 \mathrm{~kJ} \mathrm{~m}^{-3}$ and $73 \mathrm{kAm}^{-1}$; much lower than that of maghemite and magnetite. We can also calculate the average radius of the clusters to be $9 \mathrm{~nm}$. This confirms that during FCC most of the $\mathrm{Fe}$ is deposited as clusters with sizes of a few nm, big orange and black $\mathrm{Fe}$ aggregates included. Considering that, in fractions 1 and 5, when $\mathrm{Fe}$ content increases with a factor of 2 the magnetic moment increases with a factor of 13 and that FC measurements show very different behaviours at low

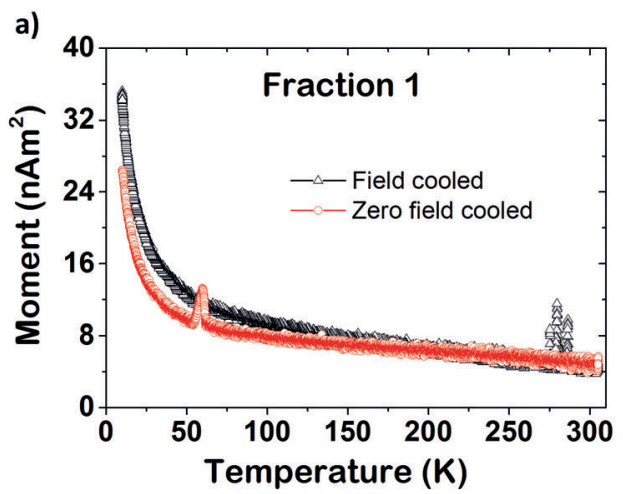

b)

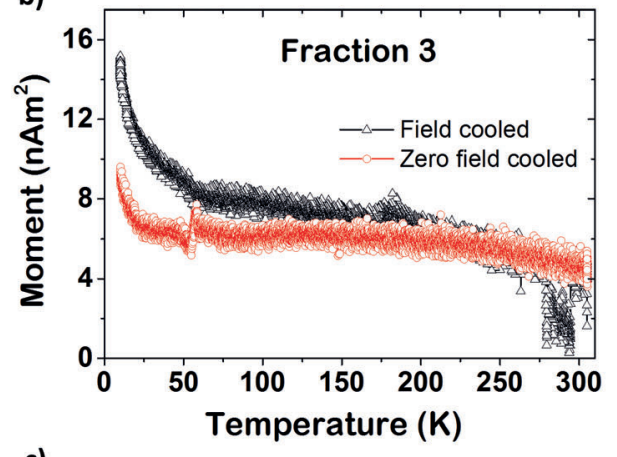

c)

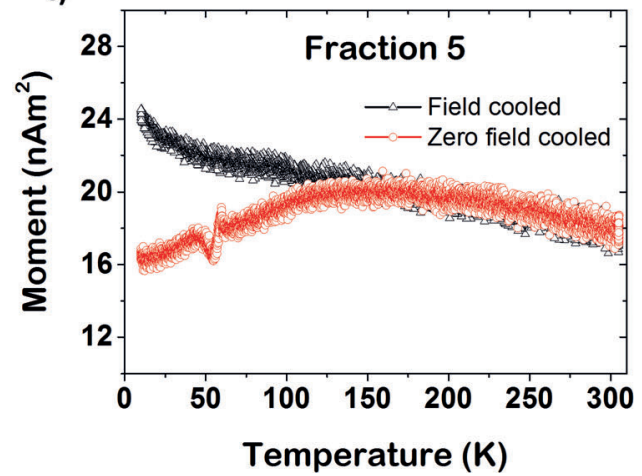

Figure 4. ZFC and FC results of sorted ECAT a) approximately 150 particles from fraction 1, b) approximately 100 particles from fraction 3 and c) approximately 500 particles from fraction 5. temperature, it is suggested that Fe deposited during FCC is in another ${ }^{[20,28]}$ and more magnetic form. The complexity and number of $\mathrm{Fe}$ (oxide) types and knowing that the magnetic moment of fractions 2-5 could be caused by a combination of these different forms only allows for a speculative explanation about its final form.

To verify whether these $\mathrm{Fe}$ clusters are related to a decrease in the amount of available Brønsted acid sites (after this referred to as acidity) as a result of pore blocking, staining experiments were performed using 4-methoxystyrene and Nile blue A. Previous work demonstrated that the Brønsted acid-catalysed oligomerization of 4-methoxystyrene visualizes zeolite domains by fluorescence micro-spectroscopy. ${ }^{[43,44,48]}$ This fluorescence was used as a measure for acidity by averaging the fluorescence intensity per particle over \pm 100 ECAT particles for all five fractions.

These average intensities were compared with Nile blue A staining results. In contrast to 4-methoxystyrene, which reacts within the zeolite, Nile blue A is a molecule that can only enter the meso- and macropores of FCC particles (Figure $5 \mathrm{a}) .{ }^{[43]}$ Therefore, it can be used as a measure of pore accessibility. It is expected that with increasing metal accumulation, both acidity and pore accessibility drop. ${ }^{[23]}$ However, when we look at the accessibility and acidity of the magnetically sorted fractions we see a different behaviour. In order to assess this in detail we performed staining experiments with 4-methoxystyrene and Nile Blue $\mathrm{A}$ in combination with fluorescence micro-spectroscopy (Figure S9).

Figure 5 shows the fluorescence intensity distribution for 4-methoxystyrene and Nile Blue A per fraction. A single particle analysis was performed by placing multiple ECAT particles in the field of view of the fluorescence microscope (Figure 5b). For each particle, an average fluorescence spectrum was measured and the emission maximum was used to create intensity histograms for both staining agents per fraction. In general, the spread within all the fractions is large, meaning that every fraction still contains very active and deactivated particles. In Figure $5 \mathrm{c}$,d, it can be seen that in fraction 1 , containing $80 \%$ of all ECAT particles, most particles show a high fluorescence signal, which can be related to high acidity. Although a very clear decrease in acidity is detected, the particles in the fractions with higher $\mathrm{Fe}$ content are not all deactivated (Figure S9). Furthermore, there is no significant change in the accessibility histograms. For example, in fraction 4, there are still ECAT particles with high acidity and high accessibility; in fact, the particles with surface Fe aggregates show very high fluorescence intensity. This means that when an aggregate of Fe is present, the rest of the particle can still be accessible for catalysis. This indicates that the presence of $\mathrm{Fe}$ aggregates does not automatically imply the presence of Fe deposited as a shell. The decrease in acidity can be caused by other deactivating processes like, for example, dealumination. ${ }^{[45]}$

In conclusion, a newly developed 3D printed microfluidic chip has been used to sort a set of ECAT particles magnetophoretically into 5 fractions, based on their magnetic moment. We believe that this technology could also be used to sort other magnetic catalysts for analysis purposes, for 

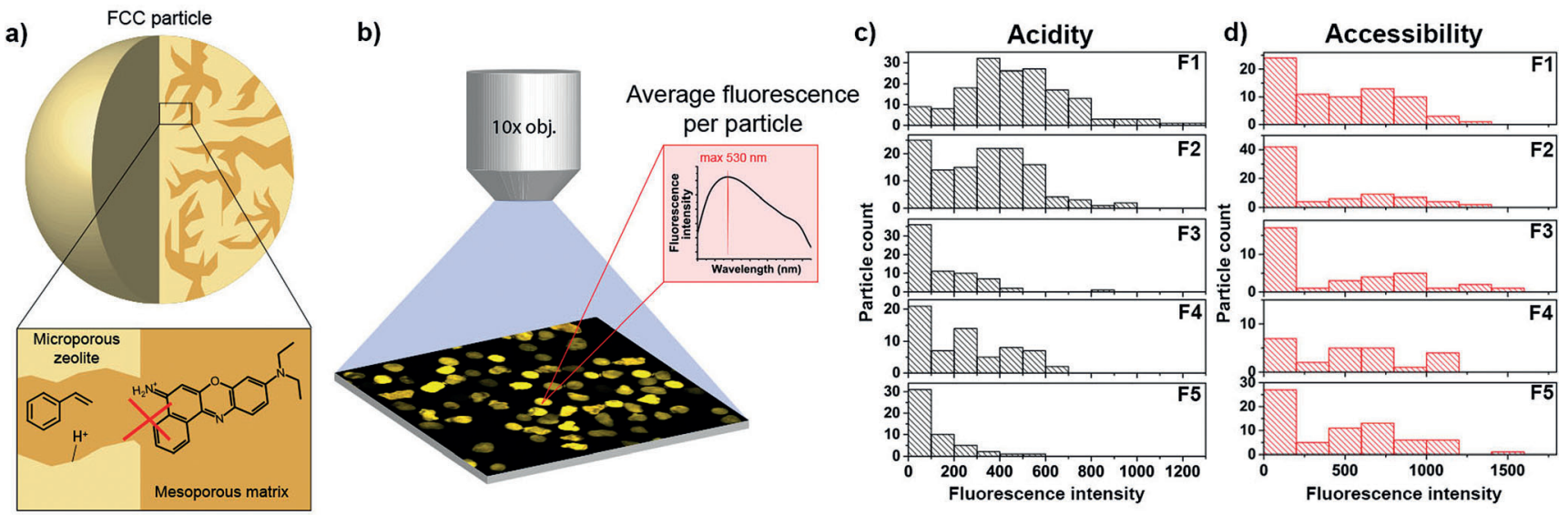

Figure 5. a) Schematic representation of Nile blue and styrene staining, indicating the zeolite shape selectivity that prevents Nile blue A from entering the zeolites' microporous structure. b) Fluorescence micro-spectroscopy images were collected and analysed per particle. Details can be found in Figure S9. Histograms of the average fluorescence intensity per ECAT particle fraction 1-5 of c) 4-methoxystyrene oligomers as a measure for acidity and d) Nile blue as a measure for pore accessibility.

example Fischer-Tropsch synthesis catalysts. It can serve as a tool to decrease the heterogeneity of to be analysed samples from an industrial FCC reactor unit or from other reactors, containing for example, Fischer-Tropsch synthesis catalysts. The trends in magnetic moment of the sorted FCC particles correlate with $\mathrm{Fe}$ content; when $\mathrm{Fe}$ content increases by a factor of 2 the magnetic moment increases by a factor of 13 . The magnetic properties of the FCC particles, extracted by a Langevin fitting show that their magnetic susceptibility does not vary between the fractions. Also, low temperature magnetization measurements show different behaviours at low temperatures, but similar blocking temperature between the fractions. These magnetic observations strongly point to the direction that variation in magnetic moment is caused by an increased number, different form but similar size of ironcontaining nano-clusters. In addition, on average, when the $\mathrm{Fe}$ content increases, the acidity generally decreases. However, the spread of acidity within each fraction is still large: not all highly magnetic particles show a high degree of deactivation.

\section{Acknowledgements}

We thank Hans de Boer (Twente University, UT), Mark Smithers (UT), Mark Huijben (UT), Hans Keizer (UT), Kristel Jagtenberg (Utrecht University, UU) and Yoni Nederstigt (UU) for their contributions. This work was supported by the Netherlands Centre for Multiscale Catalytic Energy Conversion (MCEC), an NWO Gravitation programme funded by the Ministry of Education, Culture and Science of the government of the Netherlands.

\section{Conflict of interest}

The authors declare no conflict of interest.
Keywords: fluid catalytic cracking - heterogeneous catalysis . magnetic properties - magnetophoresis - microfluidics

[1] A. A. S. Bhagat, H. Bow, H. W. Hou, S. J. Tan, J. Han, C. T. Lim, Med. Biol. Eng. Comput. 2010, 48, 999.

[2] C. Wyatt Shields IV, C. D. Reyes, G. P. López, Lab Chip 2015, 15, 1230.

[3] D. R. Gossett, W. M. Weaver, A. J. Mach, S. C. Hur, H. T. K. Tse, W. Lee, H. Amini, D. Di Carlo, Anal. Bioanal. Chem. 2010, 397, 3249.

[4] A. Valero, J. N. Post, J. W. Van Nieuwkasteele, P. M. Braak, W. Kruijer, A. Van Den Berg, Lab Chip 2008, 8, 62.

[5] R. M. Tiggelaar, F. Benito-lopez, D. C. Hermes, H. Rathgen, R. J. M. Egberink, F. G. Mugele, D. N. Reinhoudt, A. Van Den Berg, W. Verboom, H. J. G. E. Gardeniers, Chem. Eng. J. 2007, $131,163$.

[6] P. Van Male, M. H. J. M. De Croon, R. M. Tiggelaar, A. Van Den Berg, J. C. Schouten, Int. J. Heat Mass Transfer 2004, 47, 87.

[7] G. N. Doku, W. Verboom, N. Reinhoudt, A. Van Den Berg, Tetrahedron 2005, 61, 2733.

[8] H. Wensink, F. Benito-lopez, D. C. Hermes, W. Verboom, H. J. G. E. Gardeniers, N. Reinhoudt, A. Van Den Berg, Lab Chip 2005, 5, 280

[9] S. Marre, K. F. Jensen, Chem. Soc. Rev. 2010, 39, 1183.

[10] M. Tokeshi, T. Minagawa, K. Uchiyama, A. Hibara, K. Sato, H. Hisamoto, T. Kitamori, Anal. Chem. 2002, 74, 1565.

[11] T.-A. Meier, R. J. Beulig, E. Klinge, M. Fuss, S. Ohla, D. Belder, Chem. Commun. 2015, 51, 8588.

[12] K. L. A. Chan, S. Gulati, J. B. Edel, A. J. de Mello, S. G. Kazarian, Lab Chip 2009, 9, 2909.

[13] Y. H. Kim, L. Zhang, T. Yu, M. Jin, D. Qin, Y. Xia, Small 2013, 9, 3462.

[14] X. Yao, Y. Zhang, L. Du, J. Liu, J. Yao, Renewable Sustainable Energy Rev. 2015, 47, 519.

[15] E. T. C. Vogt, B. M. Weckhuysen, Chem. Soc. Rev. 2015, 44, 7342.

[16] H. S. Cerqueira, G. Caeiro, L. Costa, F. R. Ribeiro, J. Mol. Catal. A 2008, 292, 1 . 
[17] Handbook of Heterogeneous Catalysis, 2nd ed. (Eds.: G. Ertl, H. Knözinger, F. Schüth, J. Weitkamp), Wiley-VCH, Weinheim, 2008.

[18] V. Komvokis, L. X. L. Tan, M. Clough, S. S. Pan, B. Yilmaz in Zeolites in Sustainable Chemistry (Eds.: F.-S. Xiao, X. Meng), Springer, Berlin, 2016, pp. 271-297.

[19] R. Sadeghbeigi, Fluid Catalytic Cracking Handbook, Elsevier, Amsterdam, 2012.

[20] A. M. Wise, J. N. Weker, S. Kalirai, M. Farmand, D. A. Shapiro, F. Meirer, B. M. Weckhuysen, ACS Catal. 2016, 6, 2178.

[21] D. A. M. De Winter, F. Meirer, B. M. Weckhuysen, ACS Catal. 2016, $6,3158$.

[22] B. I. R. Mitchell, Ind. Eng. Chem. Prod. Res. Dev. 1980, 19, 209.

[23] F. Meirer, S. Kalirai, D. Morris, S. Soparawalla, Y. Liu, G. Mesu, J. C. Andrews, B. M. Weckhuysen, Sci. Adv. 2015, 1, e1400199.

[24] F. Meirer, D. T. Morris, S. Kalirai, Y. Liu, J. C. Andrews, B. M. Weckhuysen, J. Am. Chem. Soc. 2015, 137, 102.

[25] F. Meirer, S. Kalirai, J. N. Weker, Y. Liu, J. C. Andrews, B. M. Weckhuysen, Chem. Commun. 2015, 51, 8097.

[26] S. Kalirai, U. Boesenberg, G. Falkenberg, F. Meirer, B. M. Weckhuysen, ChemCatChem 2015, 7, 3674.

[27] Y. Liu, F. Meirer, C. M. Krest, S. Webb, B. M. Weckhuysen, Nat. Commun. 2016, 7, 12634.

[28] J. Ihli, D. Ferreira Sanchez, J. Rosh, V. Cuartero, O. Mathon, F Krumeich, C. Borca, T. Huthwelker, W.-C. Cheng, Y.-Y. Shu, et al., Angew. Chem. Int. Ed. 2017, 56, 14031; Angew. Chem. 2017, 129, 14219.

[29] J. Ihli, R. R. Jacob, M. Holler, M. Guizar-Sicairos, A. Diaz, J. C. Da Silva, D. Ferreira Sanchez, F. Krumeich, D. Grolimund, M. Taddei, et al., Nat. Commun. 2017, 8, 809.

[30] G. R. Dyrkacz, L. Ruscic, C. L. Marshall, W. Reagan, Energy Fuels 2000, 71, 849.

[31] A. K. Au, W. Huynh, L. F. Horowitz, A. Folch, Angew. Chem. Int. Ed. 2016, 55, 3862; Angew. Chem. 2016, 128, 3926.

[32] Y. Gao, Y. C. Jian, L. F. Zhang, J. P. Huang, J. Phys. Chem. C 2007, 111, 10785.

[33] N. Pamme, Lab Chip 2006, 6, 24.

[34] N. Pamme, A. Manz, Anal. Chem. 2004, 76, 7250.
[35] M. A. M. Gijs, F. Lacharme, U. Lehmann, Chem. Rev. 2010, 110, 1518.

[36] N. Pamme, C. Wilhelm, Lab Chip 2006, 6, 974.

[37] N. M. Karabacak, P. S. Spuhler, F. Fachin, E. J. Lim, V. Pai, E. Ozkumur, J. M. Martel, N. Kojic, K. Smith, P. Chen, et al., Nat. Protoc. 2014, 9, 694.

[38] E. P. Furlani, J. Appl. Phys. 2006, 99, 024912.

[39] D. Mance, J. van der Zwan, M. E. Z. Velthoen, F. Meirer, B. M. Weckhuysen, M. Baldus, E. T. C. Vogt, Chem. Commun. 2017, 53, 3933.

[40] T. L. Goolsby, H. F. Moore, Sep. Sci. Technol. 1997, 32, 655.

[41] L. R. Aramburo, S. Wirick, P. S. Miedema, I. L. C. Buurmans, F. M. F. De Groot, B. M. Weckhuysen, Phys. Chem. Chem. Phys. 2012, 14, 6967.

[42] Z. Ristanović, M. M. Kerssens, A. V. Kubarev, F. C. Hendriks, P. Dedecker, J. Hofkens, M. B. J. Roeffaers, B. M. Weckhuysen, Angew. Chem. Int. Ed. 2015, 54, 1836; Angew. Chem. 2015, 127, 1856.

[43] I. L. C. Buurmans, J. Ruiz-mart1, W. V. Knowles, D. Van Der Beek, J. A. Bergwerff, E. T. C. Vogt, B. M. Weckhuysen, Nat. Chem. 2011, 3, 862.

[44] M. M. Kerssens, A. Wilbers, J. Kramer, P. De Peinder, G. Mesu, Faraday Discuss. 2016, 188, 69.

[45] S. Kalirai, P. P. Paalanen, J. Wang, F. Meirer, B. M. Weckhuysen, Angew. Chem. Int. Ed. 2016, 55, 11134; Angew. Chem. 2016, 128, 11300.

[46] E. L. Kugler, D. P. Leta, J. Catal. 1988, 109, 387.

[47] A. S. Escobar, M. M. Pereira, R. D. M. Pimenta, L. Y. Lau, H. S. Cerqueira, Appl. Catal. A 2005, 286, 196.

[48] I. L. C. Buurmans, J. Ruiz-martínez, S. L. Van Leeuwen, D. Van Der Beek, J. A. Bergwerff, W. V. Knowles, E. T. C. Vogt, B. M. Weckhuysen, Chem. Eur. J. 2012, 18, 1094.

Manuscript received: April 27, 2018

Accepted manuscript online: July 1, 2018

Version of record online: 


\section{Communications}

Heterogeneous Catalysis

M. Solsona, A.-E. Nieuwelink, F. Meirer, L. Abelmann, M. Odijk, W. Olthuis, B. M. Weckhuysen,*

A. van den Berg** IIII-IIII

Magnetophoretic Sorting of Single Catalyst Particles
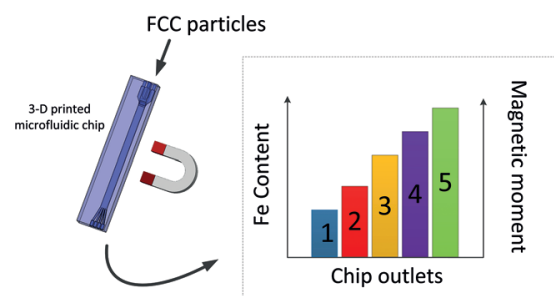

Sorted: Fluid catalytic cracking (FCC) particles can deactivate owing to $\mathrm{Fe}$ coming from the crude oil feedstock. FCC particles were magnetophoretically sorted by their magnetic moment, which correlates with Fe content. Their magnetic properties suggest that Fe deposits in small clusters on the FCC particles and that acidity decreased with increasing $\mathrm{Fe}$ content. 